\title{
Morphology-dependent resonances of a microsphere-optical fiber system
}

\author{
Giora Griffel, Stephen Arnold, Dogan Taskent, and Ali Serpengüzel* \\ Microparticle Photophysics and Photonics Laboratories, Polytechnic University of New York, Brooklyn, New York 11201 \\ John Connolly and Nancy Morris \\ David Sarnoff Research Center, Princeton, New Jersey 08543
}

Received October 16, 1995

\begin{abstract}
Morphology-dependent resonances of microspheres sitting upon an index-matched single-mode fiber half-coupler are excited by a tunable 753-nm distributed-feedback laser. Resonance peaks in the scattering spectra and associated dips in the transmission spectra for the TE and TM modes are observed. We present a new model that describes this interaction in terms of the fiber-sphere coupling coefficient and the microsphere's intrinsic quality factor $Q_{0}$. This model enables us to obtain expressions for the finesse and the $Q$ factor of the composite particle-fiber system, the resonance width, and the depth of the dips measured in the transmission spectra. Our model shows that index matching improves the coupling efficiency by more than a factor of 2 compared with that of a non-index-matched system. (c) 1996 Optical Society of America
\end{abstract}

Spherical dielectric microparticles have attracted increased attention recently ${ }^{1-4}$ because of their potential use as photonics devices. This potential stems from the combination of high- $Q$ optical resonances in a relatively small volume. These tiny cavities, whose diameters may vary from a few to several hundred micrometers, have resonances with reported $Q$ as large as $3 \times 10^{9}{ }^{1}$ Their potential applications include room-temperature persistent spectral hole-burning memories, ${ }^{2,3}$ linewidth reduction and frequency-control devices for semiconductor lasers, ${ }^{4}$ substrates for antibody and antigen detection in immunological assay, ${ }^{5}$ and microspectrum analyzers. The practical realization of these applications requires the use of solid particles in contact with a substrate from which light can be coupled into and out of the particles. We demonstrated one such mechanism recently., ${ }^{5,6}$ Morphology-dependent resonances of polystyrene microspheres immersed in water were excited upon an optical fiber half-coupler. The light source was a tunable cw dye laser, and the particles were separated from the single-mode core by $0.7 \mu \mathrm{m}$ of cladding. In this demonstration the microsphere had the appearance of resonant dust that scattered light from the fiber only at particular resonant frequencies. Soon after, another group of researchers ${ }^{7}$ used the same mechanism in air (i.e., without the surrounding liquid) and showed that the spectral width of these resonances is modified by interaction with the fiber. So far no spectrum has been presented that shows the frequency dependence for transmission through the fiber, and there is no corresponding model. In what follows we present, for the first time to our knowledge, the fiber-transmission spectra of a microsphere-fiber system that uses a tunable distributed-feedback (DFB) semiconductor laser as a light source. In addition, we present a model that describes this interaction in terms of coupling coefficients and the microsphere's intrinsic quality factor $Q_{0}$. This model enables us to obtain expressions for the $Q$ factor of the combined fiber-sphere system, the resonance width, and the depth of the dips in the transmission spectra.

The experimental setup is shown in Fig. 1. It consists of a glass microsphere sitting on top of a halfcoupler. The sphere is a commercially available BK-7 glass with a radius of $500 \pm 5 \mu \mathrm{m}$ and a sphericity of less than a half-wave. The refractive index of the sphere is 1.517 . The sphere was epoxied to a microprobe manipulator that enables one to control its relative position with respect to the polished surface of the half-coupler. The half-coupler is fabricated from an 800-nm single-mode fiber (cutoff wavelength $750 \mathrm{~nm})$, with a core radius of $1.9 \mu \mathrm{m}\left(n_{\text {core }}=1.462\right)$ and a cladding $\left(n_{\text {clad }}=1.457\right)$, which is side polished beneath the sphere to a thickness of $0.7 \mu \mathrm{m}$. The microsphere is embedded in a dielectric liquid, which is index matched to the fiber cladding. In this way we can optically eliminate the polished surface boundary, control the $Q$ factor of the fiber-sphere system, and prevent contamination of the surface of the sphere by dust particles and other residues. We excite the spherical modes by using a tunable DFB semiconductor

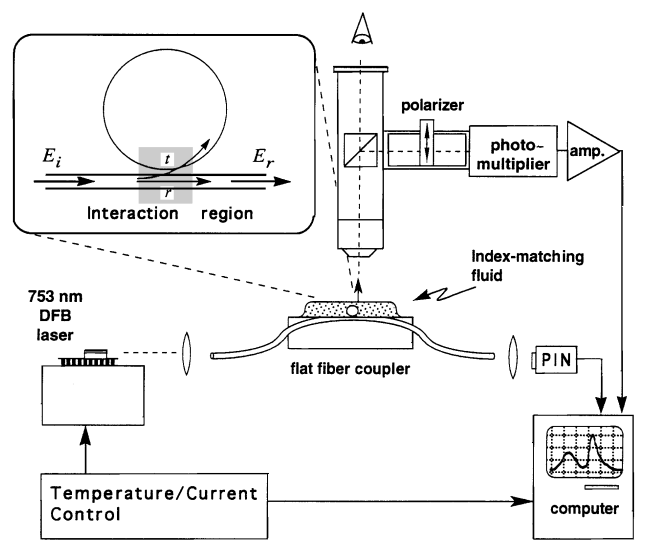

Fig. 1. Experimental setup. The inset defines the parameters $t$ and $r$.

(C) 1996 Optical Society of America 
laser whose center wavelength is $753 \mathrm{~nm}$. We achieve wavelength tuning by varying the current through the DFB, its temperature, or both. Both light transmitted through the fiber and scattered light near $90^{\circ}$ are detected and monitored. A polarizer, inserted between the microscope and the light-scattering detector, is used to separate the TE and the TM modes of the sphere. $^{4}$

Figure 2 shows the scattering and transmission spectra obtained by temperature tuning the DFB between 21.0 and $33.0^{\circ} \mathrm{C}$ at constant current, which corresponds to a wavelength range of 754.1-754.8 nm. We can clearly observe the free spectral range that includes both TE and TM modes of several orders (radial-mode functions) and the associated dips in the transmitted light. Note that modes of different order have differing linewidths (i.e., different $Q$ ). These dips were not observed in our previous experiment ${ }^{5}$ because there we used a wideband $(>22-\mathrm{GHz})$ tunable dye laser as the light source. The use of a DFB laser with a linewidth of 3-5 $\mathrm{MHz}$ enables us to couple out a substantial fraction of the source light. This feature is important for frequency control and linewidth quenching of a semiconductor laser by use of optical feedback from a fiber-sphere system. It is important to note that the fractional depth of the dips is not the same for all modes. This is due to the different radial mode functions, which results in different $Q$ factors and coupling coefficients for each of the observed modes. As we show, the coupling coefficient is one of the parameters that determine the $Q$ factor of the combined system, the magnitude of the scattered light peaks, and the transmission dips.

We repeated our experiment over a narrower region in wavelength, using current tuning. This eliminated thermal hysteresis problems associated with temperature tuning and enabled us easily to remain on a resonance for $\sim 1 \mathrm{~h}$. As shown in Fig. 3, the wavelength was varied over one free spectral range of the sphere. We show the TE mode spectrum along with the associated dips in the fiber-transmission spectrum. The TM spectrum is similar to the TE spectrum and is red shifted by $0.012 \mathrm{~nm}$. Wavelength tuning is produced by the drive current, which explains the increase in transmitted power relative to wavelength. Normalizing the transmission spectra, using data taken with the same system without the sphere, reveals that the relative depth of the transmission dips is maintained among modes of the same order ${ }^{8}$ (i.e., modes that have the same number of peaks in the radial mode function).

To analyze the scattering and transmission spectra we use the simplified two-dimensional model shown in the inset of Fig. 1. $E_{i}$ is the field in the fiber to the left of the sphere, and $E_{r}$ is the field remaining in the fiber beyond the fiber-sphere interaction region. We assume that the field coupling coefficient from the fiber to the sphere is $t$ and that the single-pass ratio between the field propagating in the fiber after the region of coupling to the sphere to that before coupling is $r$. One can show, using the coupled-mode formalism and the power-conservation relation, that $t$ is purely imaginary and that $r^{2}-t^{2}=1$. We assume that a single mode propagating in the fiber is coupled to one of the resonant modes of the sphere, whose propagation constant is given by $\beta=\beta_{0}-i \alpha$. Here $\alpha$ represents attenuation of the spherical mode as the result of leakage. If the intrinsic quality factor of the isolated sphere is given by $Q_{0}$, then the decay constant $\alpha$ is related to $Q_{0}$ by $\alpha=\beta_{0} / 2 Q_{0}$. Using these definitions, and assuming lossless material, we find the relation between $E_{r}$ and $E_{i}$ :

$$
\frac{E_{r}}{E_{i}}=\frac{r-\exp \left[-\left(\alpha+i \beta_{0}\right) L_{a}\right]}{1-r \exp \left[-\left(\alpha+i \beta_{0}\right)\right]}
$$

where $L_{a}=2 \pi a$ is the circumference of the sphere. The relation of the scattered field intensity to the incident intensity, $I_{S} / I_{i}=1-I_{r} / I_{i}=1-\left|E_{r} / E_{i}\right|^{2}$, is found after some manipulation of these relations to be

$$
\frac{I_{S}}{I_{i}}=\frac{T_{m}}{1+\frac{4 r \exp \left(-\alpha L_{a}\right)}{\left[1-r \exp \left(-\alpha L_{a}\right)\right]^{2}} \sin ^{2}\left(0.5 \beta_{0} L_{a}\right)} .
$$

Equation (2) is analogous to the expression obtained for a linear Fabry-Perot resonator with unequal mirrors. With this model the mode spacing is $\Delta \nu=$ $c /(2 \pi a n)$, where $n$ is the effective refractive index of the spherical mode participating in the coupling process and the finesse is given by

$$
F=\pi \frac{\left[r \exp \left(-\alpha L_{a}\right)\right]^{1 / 2}}{1-r \exp \left(-\alpha L_{a}\right)} .
$$

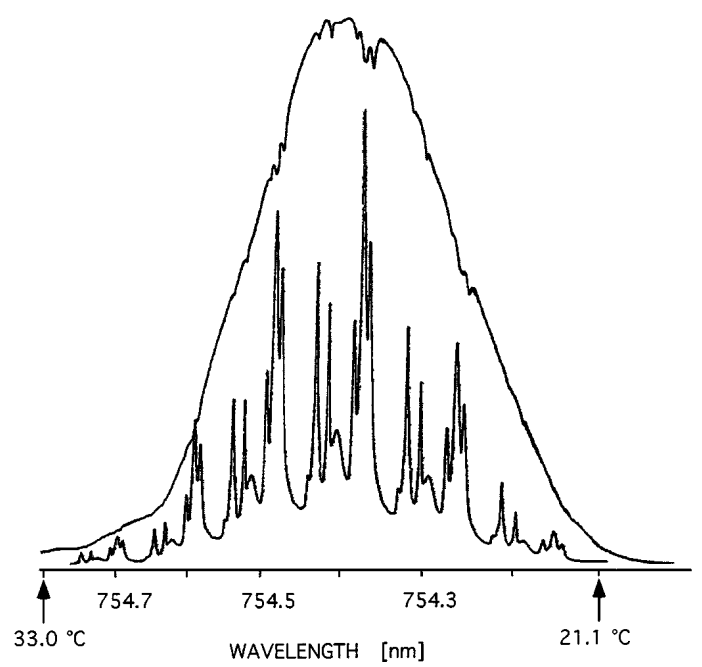

Fig. 2. Temperature-tuning spectra of both the transmission (upper curve) and right angle scattering from the sphere-fiber system.

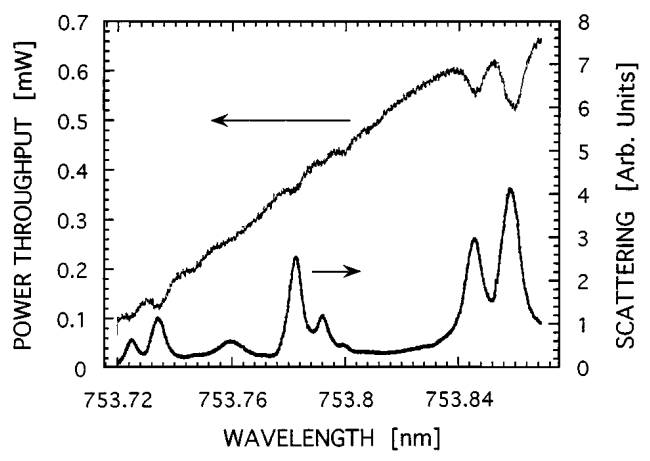

Fig. 3. Current tuning scattering and transmission spectra of the TE modes spanning one free spectral range. 
$T_{m}$, the magnitude of the resonance peaks and the transmission dips, is given by

$$
T_{m} \equiv \frac{\left(1-r^{2}\right)\left[1-\exp \left(-2 \alpha L_{a}\right)\right]}{\left[1-r \exp \left(-\alpha L_{a}\right)\right]^{2}} .
$$

From Eq. (2) and the definition of $\alpha$ we can derive the quality factor of the composite fiber-sphere system in terms of $Q_{0}$ and $r$ :

$$
Q_{T}=0.5 \beta_{0} L_{a} \frac{\sqrt{r} \exp \left(-\frac{\beta_{0}}{4 Q_{0}} L_{a}\right)}{1-r \exp \left(-\frac{\beta_{0}}{2 Q_{0}} L_{a}\right)} .
$$

Making use of the fact that $\left(\beta / 2 Q_{0}\right) L_{a} \ll 1$ (corresponding to low round-trip loss), and assuming that $|t|^{2} \ll 1$, we finally obtain the dependence of the quality factor of the composite fiber-sphere system on the sphere's intrinsic $Q_{0}$ and on a newly defined quality factor for fiber coupler $Q_{f}$ :

$$
\frac{1}{Q_{T}}=\frac{1}{Q_{0}}+\frac{1}{Q_{f}}
$$

where the contribution of the fiber coupling $Q_{f}$ is $(2 \pi X) /|t|^{2}$, with $X$, the sphere size parameter, given by $X \equiv(2 \pi a n) / \lambda$. We can arrive at the same expression for $Q_{f}$, the quality factor associated with fiber coupling, by considering the energy-loss rate from an otherwise lossless spherical oscillator in the presence of a fiber. The fraction of energy loss per circumnavigation is $|t|^{2}$. Because one circumnavigation comprises $X$ oscillations, the fractional loss per oscillation is $|t|^{2} / X$. With the definition of $Q$ as $2 \pi$ divided by the fractional energy loss per oscillation, Eq. (6) arises.

For our experiment the width of the dips in transmission spectra was independent of the separation between the particle and the fiber, although the amplitude of the dips decreased markedly as the sphere was withdrawn from the half-coupler. We conclude, based on Eq. (5), that $Q_{f} \gg Q_{0}$. In another recent experiment, for a similar glass sphere in air, the linewidth was dominated by the fiber-sphere interaction. ${ }^{6}$ Based on the data in Ref. 6, $Q_{f}$ is approximately $1 / 20$ of $Q_{0}$ when the sphere is in contact with the halfcoupler. In what follows, we show that this distinct reversal in the ratio of $Q_{f}$ to $Q_{0}$ is a consequence of the lower $Q_{0}$ in our case, which is due to the reduced optical confinement caused by the lower relative refractive index between the sphere and the surrounding liquid.

In our case $Q_{T} \sim Q_{0}$. Using Eqs. (2) and (3), we find that a dip of $20 \%$ (corresponding to the maximum coupling) in the transmission spectra corresponds to $|t|^{2}=1.4 \times 10^{-2}$, which means that $1.4 \%$ of the incident power is coupled to a resonance in a single pass. This value is consistent with our original assumption; using $|t|^{2}=1.4 \times 10^{-2}$ in Eq. (6) leads to $Q_{f}=2.8 \times 10^{6}$, which is much larger than $Q_{T}$ obtained from the measured linewidth $\left(2.5 \times 10^{5}\right)$. For the experiment in Ref. 6 it was noted that a $60-\mathrm{MHz}$ increase in width resulted from placing the sphere in contact with the fiber. One can use this linewidth in estimating $|t|^{2}$, using Eq. (6). On this basis we arrive at $Q_{f}=$ $6.4 \times 10^{6}$ and $|t|^{2}=0.6 \times 10^{-2}$. We attribute our larger coupling coefficient to index matching. Index matching extends the fiber-sphere interaction range by allowing the electromagnetic field to penetrate over a larger distance on the sphere side of the polished cladding surface.

We have presented the fiber-transmission spectra of a microsphere-fiber system that uses a tunable DFB semiconductor laser as a light source. In addition we described a model for this interaction in terms of coupling coefficients and the microsphere's intrinsic quality factor, $Q_{0}$. Using this model, we obtained expressions for the $Q$ factor of the combined particlefiber system, the resonance width, and the depth of the dips measured in the transmission spectra. We reduced the problem of describing the fiber-sphere interaction to one parameter, $t$.

We are grateful for support for this research by U.S. Air Force Research Office grant F49620-94-0195. G. Griffel was supported by National Science Foundation grants ECS9308126 and 9311204.

*Present address, Department of Physics, Bilkent University, Ankara 06533, Turkey.

\section{References}

1. L. Collot, V. Lefévre-Seguin, M. Brune, J. M. Raimond, and S. Haroche, Europhys. Lett. 23, 327 (1993).

2. S. Arnold, C. T. Liu, W. B. Whitten, and J. M. Ramsey, Opt. Lett. 16, 420 (1991).

3. S. Arnold, J. Camunale, W. B. Whitten, J. M. Ramsey, and K. A. Fuller, J. Opt. Soc. Am. B 9, 819 (1992).

4. G. Griffel, A. Serpengüzel, and S. Arnold, 1995 IEEE Symposium on Frequency Control (Institute of Electrical and Electronics Engineers, New York, 1995).

5. A. Serpengüzel, S. Arnold, and G. Griffel, Opt. Lett. 20, 654 (1995).

6. S. Arnold, A. Serpengüzel, and G. Griffel, in Guided Wave Optoelectronics, T. Tamir, G. Griffel, and H. Bertoni, eds. (Plenum, New York, 1995), pp. 495-497.

7. N. Dubreuil, J.C. Knight, D. K. Leventhal, V. Sandoghdar, J. Hare, and V. Lefévre, Opt. Lett. 20, 813 (1995).

8. P. W. Barber and R. K. Chang, eds., Optical Effects Associated with Small Particles (World Scientific, Singapore, 1988), Chap. 1. 